\title{
Retroperitoneal Unicentric Castleman's Disease
}

\author{
Sertaç DEMIREL, ${ }^{1(1)}$ Tolga ALIYAZICIOĞLU, ${ }^{1}$ (D) Zeynep TOSUNER ${ }^{2}$
}

'Department of General Surgery, Şişli Memorial Hospital, Istanbul-Turkey

${ }^{2}$ Department of Pathology, Atakent Acıbadem Hospital, Istanbul-Turkey

\section{Dear Editor,}

Castleman's disease (CD) is an unusual lymphoproliferative disorder first described in 1954 by Benjamin and Virginia.[1] The etiology of the disease is not clear, however, the role of chronic inflammation, immunosuppression, disruption in the production of interleukin 6, and human herpesvirus 8 has been demonstrated. Histologically, there are four subtypes; hyaline vascular, plasma cell variant, multicentric (mixed), and plasmablastic variant.[2] Hyaline vascular CD is characterized by prominent vascular proliferation and hyalinization of the vessel walls. Lymphoid follicles have atretic germinal centers traversed by penetrating vessels that are called "lollipop follicle." Thickened mantle zones consisting of lymphocytes arranged in layers-onion skin appearance are present throughout the lymph node. Fused mantle zones that contain more than one germinal center can be observed. Extensive vascular proliferation with perivascular hyalinization is evident in the interfollicular areas.[3,4] There are two subtypes clinically, unicentric CD (UCD) and multicentric CD (MCD). UCD typically presents as singular enlarged of lymph node. About $70 \%$ of all CD patients are in this group. UCD is often found in both males and females aged 20-30 years and presents asymptomatically.[5] MCD is a widespread disease associated with systemic symptoms.[6] Patients of the UCD usually have a good prognosis and are treated with surgical excision.[6] MCD tends to behave aggressively, similarly to lymphoma, and is treated with immunotherapy, chemotherapy, and radiotherapy.[7-9] CD most commonly develops in the mediastinum and the second most common localization cervical region. Retroperitoneal localization is extremely rare.[10]
An asymptomatic 35-year-old man received a routine examination in Memorial Sisli Hospital (Istanbul, Turkey). He had no feature in his medical history. A physical examination did not reveal any abnormal results and the patient's full blood count and biochemical profile were normal. Abdominal ultrasound examination revealed a $70 \mathrm{~mm} \times 50 \mathrm{~mm}$ solid hypoechoic mass lesion adjacent to the left lateral wall of the bladder. Abdominal contrast-enhanced computerized tomography revealed a $6.6 \mathrm{~cm} \times 5.2 \mathrm{~cm} \times 3.6 \mathrm{~cm}$ solid mass adjacent to internal iliac vessels and causing indentation left lateral wall of bladder. Due to the localization of the mass and possibility of extragonadal germ cell tumor, alpha-fetoprotein, lactate dehydrogenase, and beta human chorionic gonadotropin hormone values were requested and all results were within normal ranges. It was decided to surgically removal of the solid mass for a definitive diagnosis. Under general anesthesia, an upper midline incision was made and the peritoneal cavity was entered. Generalized abdominal exploration revealed only a solid mass below the left ureter and under the left iliac vessels. The solid mass freed up surrounding tissues. Sacral plexus branches were seen behind the solid mass. The solid mass was totally removed. The solid mass was a nodular structure, measuring $71 \mathrm{~g}, 6 \mathrm{~cm} \times 5.5 \mathrm{~cm} \times 4.5 \mathrm{~cm}$ in size. The patient was discharged on the $2^{\text {nd }}$ post-operative day. Histologic sections showed a lymph node with a preserved basic structure containing fibrous septae. Lymphoid follicles with one or more germinal centers, follicles with inconspicuous or regressed germinal centers, and follicles with thickened mantle zones were distributed within the lymph node. Small lymphoid cells were seen in the interfollicular area. Dilated congested vessels were observed in perinodal adipose tissue. No evidence 
of specific inflammation, event, or malignancy was found. Pathological examination consistent with hyaline vascular type of Castleman's disease. Ultrasonography (USG) demonstrates homogeneous hypoechoic mass and computed tomography (CT) demonstrates a homogeneous mass with non-specific microcalcifications. USG and CT are not diagnostic as seen in our case.[9] Although magnetic resonance imaging (MRI) is superior to CT as it shows soft-tissue better, it is not diagnostic for $\mathrm{CD}$. Pre-operative fine-needle aspiration cytology has no role in diagnosis due to its low specificity. Therefore, a definitive diagnosis can only be made by pathological examination of the removed mass. Treatment methods may vary between unicentric and MCD. Standard treatment for UCD is en bloc surgical removal as performed in our case. Five-year survival rate after surgery is almost $100 \%$. Recurrences are mainly reported in cases where surgical removal cannot be performed completely.[11] Cytoreduction of radiotherapy has also been advocated in cases where complete resection is not feasible.[12] There is no curative treatment for MCD. Corticosteroids, immunosuppressive drugs, chemotherapy, and radiotherapy have been tried for the treatment of MCD. $[9,12,13]$ Various rare benign tumors, primary or metastatic malignant tumors, can be seen in the retroperitoneum. CD should always be considered in the differential diagnosis of hypervascular masses detected in the retroperitoneum. Complete surgical removal of the mass is the most effective treatment for retroperitoneal UCD.

\section{References}

1. Benjamin C, Virginia WT. CASE records of the Massachusetts general hospital weekly clinicopathological exercises: Case 40011. N Engl J Med 1954;250(1):26-30.
2. Saeed-Abdul-Rahman I, Al-Amri AM. Castleman disease. Korean J Hematol 2012;47(3):163-77.

3. Wu D, Sihia M, Jaffe ES. Pathology of castleman disease. Hematol Oncol Clin N Am 2018;32(1):37-52.

4. Wang HW, Pittaluga S, Jaffe ES. Multicentric castleman disease, where are we now? Semin Diagn Pathol 2016;33(5):294-306.

5. Shah D, Darji P, Lodha S, Bolla S. Unicentric Castleman's disease of abdomen. J Radiol Case Rep 2013;7(3):26-33.

6. Bucher P, Chassot G, Zufferey G, Ris F, Huber O, Morel P. Surgical management of abdominal and retroperitoneal Castleman's disease. Worl J Surg Oncol 2005;3:33.

7. Guo H, Shen Y, Wang WL, Zhang M, Li H, Wu YS, et al. Castleman diseasemimicked pancreatic carcinoma: report of two cases. World J Surg Oncol 2012;10:154.

8. Shimokiharaa K, Kawaharab T, Kasaharaa R, Kasugaa J, Sugiuraa S, Tajiric R, et al. Retroperitoneal Castleman's disease. Case Rep Oncol 2019;12(3):885-9.

9. Kim TJ, Han JK, Kim YH, Kim TK, Choi BI. Castleman disease of theabdomen: İmaging spectrum and clinicopathologic correlations. J Comput Assist Tomogr 2001;25(2):207-14.

10. Nakata K, Iwahashi N, Matsukawa H, Noguchi T, Yahata A, Ota N, et al. Laparoscopically resected Castleman's disease in the pelvic retroperitoneum: A case report. Mol Clin Oncol 2020;12(2):169-73.

11. Shahidi H, Myers JL, Kvale PA. Castleman's disease. Mayo Clin Proc 1995;70(10):969-77.

12. Roca B. Castleman's disease. A review. AIDS Rev 2009;11(1):3-7.

13. Horster S, Jung C, Zietz C, Cohen CD, Siebeck M, Goebel FD. AIDS, multicentric Castleman's disease, and plamablastic leukemia: Report of along-term survival. Infection 2004;32(5):296-8. 